\title{
Effect of ketorolac on the prevention of emergence agitation in children after sevoflurane anesthesia
}

\author{
Deokkyu Kim, A Ram Doo, Hyungsun Lim, Ji-Seon Son, Jun-Rae Lee, Young-Jin Han, and Seonghoon Ko \\ Department of Anesthesiology and Pain Medicine, Chonbuk National University Medical School and Hospital, Jeonju, Korea
}

Background: The purpose of this study was to evaluate the effects of ketorolac on the incidence and severity of emergence agitation in children recovering from sevoflurane anesthesia.

Methods: Eighty-five children aged 3 to 7 years were randomly assigned to the control group or the ketorolac group ( $1 \mathrm{mg} / \mathrm{kg}$ ketorolac). The children were evaluated by the Pediatric Anesthesia Emergence Delirium Scale and a fourpoint agitation scale.

Results: The median agitation scores did not differ significantly between the two groups. The overall incidence of emergence agitation was similar in the two groups ( $41 \%$ in the control group vs. $32 \%$ in the ketorolac group, $\mathrm{P}=$ 0.526). The number of children who received rescue drugs for treatment of emergence agitation was not significantly different between the two groups.

Conclusions: The administration of $1 \mathrm{mg} / \mathrm{kg}$ of ketorolac is not effective in decreasing the incidence and severity of emergence agitation in children aged 3 to 7 years after sevoflurane anesthesia. (Korean J Anesthesiol 2013; 64: 240-245)

Key Words: Agitation, Anesthesia, Ketorolac, Pediatrics.

\section{Introduction}

Emergence agitation (EA) or delirium is a well-recognized phenomenon in children during the immediate postanesthesia period. This phenomenon is characterized by behaviors during the recovery period that include thrashing, kicking, restlessness, inconsolable crying, disorientation, delusion, hallucination and impairments in cognitive thinking and memory [1]. Combative behaviors like thrashing and kicking are more common than simple restlessness and incoherence in children with EA, which can increase the risk of adverse effects including falling from the bed, increased postsurgical bleeding and pain, contamination of the surgical wound, and inadvertent removal of drains and intravenous catheters [2,3]. Therefore, EA is considered as a potentially serious complication in spite of its spontaneous resolution. Although the pathophysiology remains unclear, several risk factors have been associated with an increased incidence of EA, including age and temperament of the child, surgical procedures, anesthetic agents, and preoperative anxiety. Several studies have confirmed that the incidence of

Received: August 6, 2012. Revised: 1st, August 28, 2012; 2nd, September 10, 2012. Accepted: September 20, 2012.

Corresponding author: Seonghoon Ko, M.D., Department of Anesthesiology and Pain Medicine, Chonbuk National University Hospital, 634-18, Keumam-dong, Dukjin-gu, Jeonju 561-712, Korea. Tel: 82-63-250-1979, Fax: 82-63-250-1240, E-mail: shko@jbnu.ac.kr

(c) This is an open-access article distributed under the terms of the Creative Commons Attribution Non-Commercial License (http:// creativecommons.org/licenses/by-nc/3.0/), which permits unrestricted non-commercial use, distribution, and reproduction in any medium, provided the original work is properly cited. 
EA is increased following sevoflurane anesthesia [4-6]. The prevalence of EA after sevoflurane anesthesia varies between $20 \%$ and $80 \%$ depending on the criteria to define EA and the monitored time interval after emergence from anesthesia $[5,7]$.

It is difficult to differentiate EA from behaviors related to postoperative pain because a child's reactions to postoperative pain are similar to that of EA. However, several studies showed that the incidence of EA in painful procedures is similar to in nonpainful procedures $[2,3,8]$. These results support the conclusion that pain does not contribute to EA. Nevertheless, a number of analgesics including fentanyl have been reported to reduce the incidence of EA to varying degrees [9-11]. The analgesic must provide adequate analgesia and minimize sideeffects, particularly in children undergoing ambulatory surgery. Ketorolac tromethamine, a nonsteroidal anti-inflammatory drug (NSAID), has been proposed as an alternative choice of analgesic in children undergoing surgery $[12,13]$.

Although ketorolac is one of the most widely used analgesics in children undergoing surgical procedures, there are few studies that have investigated the effects of ketorolac on EA. The purpose of this study was to evaluate the effects of ketorolac on the incidence and severity of EA in children recovering from sevoflurane anesthesia.

\section{Materials and Methods}

This prospective, randomized, double-blinded, controlled study was approved by the relevant Institutional Review Board. Eighty-five children aged 3 to 7 years, American Society of Anesthesiologists physical status I or II, undergoing elective surgical procedure were enrolled. Informed written consent to participate in the study was obtained from the parents. Children with a medical history of cardiovascular or renal disease, neurologic impairment, developmental delay, sensitivities to ketorolac, or anxiety disorder were excluded from participation. Subjects were randomly assigned to either the control group or the ketorolac group by computer-generated random number.

Patients were admitted the evening before surgery. The research team anesthesiologist visited the pediatric ward to conduct preoperative interviews and measure baseline anxiety score. Children's preoperative anxiety was determined by the modified Yale Preoperative Anxiety Scale (mYPAS) after group assignment. The mYPAS contains 22 items in five categories qualifying anxiety in young children. The score range is 23.3100 , with a score $\leq 30$ indicating the subject does not have anxiety and a score $>30$ indicating subject anxiety [14]. After consent was obtained, demographic data were collected from the parents and the patient's medical charts. All surgeries were performed in the morning sessions. A $24 \mathrm{G}$ catheter was inserted into a peripheral vein the night before surgery or the morning of surgery after eutectic mixture of local anesthetics (EMLA) cream application, and the time was recorded. A balanced salt solution was administered according to standard fluid administration guidelines. No premedication was administered and the children were accompanied by their parent and a research team member during the transfer to the operating room. After monitor placement with ECG and pulse oximetry, the final anxiety score using mYPAS was taken just before anesthesia induction. A parent was permitted to be present during induction of anesthesia.

Standard anesthetic regimens and techniques were used for all patients. Intravenous anesthetic induction was performed using 4-6 $\mathrm{mg} / \mathrm{kg}$ thiopental and patient blood pressure was noninvasively monitored with a Zeus ${ }^{\circledR}$ anesthesia workstation (Dräger Medical, Lübeck, Germany). For facilitation of tracheal intubation, the patients received $0.6 \mathrm{mg} / \mathrm{kg}$ rocuronium. Anesthesia was maintained with $50 \%$ nitrous oxide in oxygen and sevoflurane. End tidal carbon dioxide partial pressure was maintained at 30-35 mmHg. Sevoflurane was administered to maintain an end-tidal sevoflurane concentration of 1.5$3.0 \%$ according to blood pressure and heart rate. Patients were administered either $1 \mathrm{mg} / \mathrm{kg}$ of ketolorac (ketorolac group) diluted to $2 \mathrm{ml}$ or $2 \mathrm{ml}$ saline (control group) intravenously before end of the surgical procedure. The anesthesiologists were blinded to group assignment. Ketorolac and placebo were prepared in the anesthesia preparation room by one research member. At the end of surgery, sevoflurane and nitrous oxide administration was discontinued, and the patients received pyridostigmine and glycopyrrolate for reversal of the neuromuscular blockade. The trachea was extubated and the children were transferred to the postanesthesia care unit (PACU) in a quiet and warm environment without any stimulus. The time to extubation, defined as time from the end of anesthetic administration to tracheal extubation, was recorded.

In the PACU, monitoring consisted of ECG, respiratory rate, and peripheral oxygen saturation $\left(\mathrm{SpO}_{2}\right)$. To avoid physical stimulation, noninvasive blood pressure (NIBP) was not measured. Heart rate, respiratory rate, and $\mathrm{SpO}_{2}$ were recorded every $5 \mathrm{~min}$ for the first $30 \mathrm{~min}$, then at 10-minute intervals for the next hour. Parents were allowed to join their children in the PACU. The anesthesiologist, data-collecting nurse, and child's parents were blinded to group assignment. Postoperative pain was assessed by the anesthesiologist. If a child complained of pain or exhibited signs or symptoms of pain, intravenous fentanyl $1 \mu \mathrm{g} / \mathrm{kg}$ was administered. The administration of fentanyl as a rescue analgesic was recorded. The primary endpoint of the study was the incidence of EA during the first hour after awakening. The children were evaluated at 5 and 20 min after PACU arrival by a four-point agitation scale (FPAS), in which 1 = quiet and calm child, 2 = crying but consolable child, 
3 = moderately agitated child evident by screaming and crying, and 4 = aggressive child who must be physically restrained to avoid harm $[9,15,16]$. Also, the children were evaluated at 20 min after PACU arrival by the Pediatric Anesthesia Emergence Delirium Scale (PAEDS) [1] because PAEDS in children who are asleep is inappropriate. Besides 5 and 20 min, EA scales were measured at any time when a child showed behavioral changes in the PACU. We defined EA was defined as a FPAS of 3 or 4 , or a PAED score $\geq 10$. The highest scores recorded at any time were used to determine presence of agitation and to compare between the two groups. If agitation persisted more than $5 \mathrm{~min}$, children were treated with $1 \mathrm{mg} / \mathrm{kg}$ propofol at the discretion of the attending anesthesiologist and observed to avoid respiratory depression. The number and time of rescue drug administration was recorded.

\section{Sample size determination and statistical analyses}

In order to determine the appropriate sample size, the incidence of EA in children after sevoflurane anesthesia was assumed to be $65 \%$. A sample size calculation determined by proportions sample size that 42 children per group were required $(\alpha=0.05, \beta=0.2)$ to detect a $50 \%$ reduction in the incidence of EA with ketorolac.

Data were analyzed using SigmaStat 3.5 (Systat Software, San Jose, CA, USA), and are presented as a number (n), percentage $(\%)$, mean $\pm \mathrm{SD}$, or median as appropriate. Student t-test was used to compare the mean value of quantitative data between the two groups. Nonparametric data such as PAED score and FPAS were compared between groups with the Mann-Whitney U test. Categorical data were compared by the chi-squared test or Fisher Exact test where appropriate, and included the incidence of EA and the number of patients requiring rescue drugs, expressed as a number or percentage. A P value $<0.05$ was considered statistically significant in all tests.

Table 1. Patient Characteristics

\begin{tabular}{lccc}
\hline & Control group & Ketorolac group & P values \\
\hline No. of patients & 42 & 43 & \\
Age (yr) & $4.9 \pm 1.4$ & $4.6 \pm 1.3$ & 0.309 \\
Height (cm) & $112.0 \pm 9.0$ & $111.8 \pm 11.5$ & 0.929 \\
Weight (kg) & $21.8 \pm 9.6$ & $20.4 \pm 5.5$ & 0.410 \\
Gender (M/F) & $28 / 14$ & $27 / 16$ & 0.883 \\
Fasting time (hr) & $11.4 \pm 1.9$ & $11.7 \pm 1.9$ & 0.469 \\
Anesthesia time (min) & $68.0 \pm 21.4$ & $67.8 \pm 22.8$ & 0.967 \\
Surgery & & & 0.988 \\
$\quad$ Tonsillectomy & 20 & 21 & \\
Herniorrhaphy & 11 & 12 & \\
Hand surgery & 4 & 4 & \\
Other & 7 & 6 & \\
\hline
\end{tabular}

Data are expressed as mean \pm SD except the number of patients and the types of surgery.

\section{Results}

Eighty of the 85 enrolled children completed the study. Two children were excluded because of cancellation of operation and three children were excluded because of a study protocol violation. There were 39 patients in the control group and 41 in the ketorolac group. The two study groups were comparable with respect to demographic data, fasting time, duration of anesthesia, and type of surgery (Table 1). No adverse hemodynamic or respiratory complications were noted during the study. There was no significant difference in the median preoperative anxiety score using mYAS between two groups in the pediatric ward (median: 23.3 and range: $23.3-23.3$ in the control group vs. median 23.3 and range: $23.3-30.0$ in the ketorolac group, $\mathrm{P}=0.178$ ) and in the operating room (median: 31.7 and range: $25.0-41.7$ in the control group vs. median: 31.7 and range: $27.5-47.5$ in the ketorolac group, $\mathrm{P}=0.447$ ). The time from the end of sevoflurane administration to extubation was similar in the two groups $(8.3 \pm 2.9$ vs. $7.5 \pm 2.3 \mathrm{~min}, \mathrm{P}=0.174)$.

There was no significant difference in the incidence of EA between control groups and the ketorolac group at $5 \mathrm{~min}$ (one patient, $3 \%$ vs. four patients, $10 \%, \mathrm{P}=0.360)$ and at $20 \mathrm{~min}(16$ patients, $41 \%$ vs 11 patients, $27 \%, \mathrm{P}=0.269)$. The total number of patients displaying EA was $16(41 \%)$ in the control group and $13(32 \%)$ in the ketorolac group ( $\mathrm{P}=0.526)$ (Fig. 1). The median agitation scores using the PAEDS and FPAS were not significantly different between the two groups (Table 2). The number of children who received propofol as a rescue drug for treatment of EA was not significantly different between the two groups (5/39 in the control group, 4/41 in the ketorolac group, $\mathrm{P}$ $=0.734$ ). No patient showed EA after $30 \mathrm{~min}$ in the PACU. There was a significant difference in the number of children who received fentanyl for analgesia after $20 \mathrm{~min}$ in the PACU when comparing the control group $(13 / 39,33 \%)$ to the ketorolac

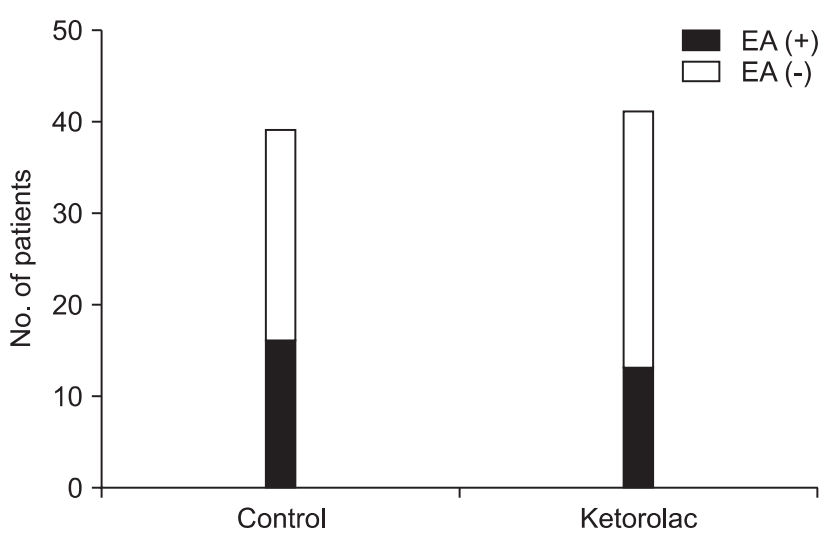

Fig. 1. The number of patients who showed emergence agitation (EA) was $16(41 \%)$ and $13(32 \%)$ in the control and ketorolac groups, respectively. There was no significant difference between two groups. 
Table 2. Pediatric Anesthesia Emergence Delirium Scale (PAEDS) and Four-point Agitation Scale (FPAS)

\begin{tabular}{llll}
\hline & & $\begin{array}{c}5 \text { min after } \\
\text { PACU arrival }\end{array}$ & $\begin{array}{c}\text { 20 min after } \\
\text { PACU arrival }\end{array}$ \\
\hline \multirow{2}{*}{ PAEDS } & Control group & & $7.0(5.0-10.0)$ \\
& Ketorolac group & & $6.0(4.0-10.0)$ \\
& Control group & $1.0(1.0-2.0)$ & $2.0(1.0-3.0)$ \\
& Ketorolac group & $1.0(1.0-2.0)$ & $2.0(1.0-2.0)$ \\
\hline
\end{tabular}

Data are expressed as median and interquartile range. PACU: postanesthesia care unit.

$\operatorname{group}(5 / 41,12 \%)(\mathrm{P}=0.046)$.

\section{Discussion}

EA is a complex phenomenon, the etiology of which is currently unknown. There is a wide variability in the incidence and behaviors of EA in different studies. Although there is no clear pathophysiologic explanation for EA, age, and anesthetic agents are thought to be important contributing factors. In general, younger children are more likely to present EA upon recovery from anesthesia [2]. Children aged 2-5 years seem to be the most vulnerable to postoperative behavioral changes [17]. Although $74 \%$ of children were $\leq 5$ years-of-age, the incidence of EA was not too high in this study. The higher incidence of EA in younger children may be related to psychological immaturity and less ability to cope in a strange environment upon awakening from general anesthesia [15]. Multiple studies have demonstrated that the incidence of EA is increased after anesthesia with an agent having a relatively lower blood/gas partition coefficient, such as sevoflurane and desflurane, and leads to rapid emergence [5,6,8-10]. Rapid emergence from anesthesia in conjunction with psychological immaturity has been a postulated cause for EA [15]. Sevoflurane anesthesia, in addition to many other factors, has been identified as a contributor to EA, and some studies have indicated that as high as $80 \%$ of children who are administered sevoflurane also exhibit EA $[2,5,18]$. Although the authors did not compare the incidence of EA in different volatile anesthetics, the incidences of EA in this study ( $41 \%$ in the control group and $32 \%$ in the ketorolac group) were comparable to previous studies, which showed lower incidence in sevoflurane anesthesia [5,7]. This lower incidence of EA compared to previous studies may be caused by intravenous induction with thiopental.

Previous studies described children's behaviors with EA as screaming, thrashing, kicking, moving arms non-purposefully, arching of the back, tilting the head backward, and staring [19-21]. In the present study, kicking and inconsolable screaming were common behaviors in children with EA, which is similar to previous studies. Malarbi et al. [21] reported that the duration of EA is usually between 5 and $25 \mathrm{~min}$. In the present study, children were evaluated at 5 and $20 \mathrm{~min}$ after arrival PACU and no child displayed EA after $30 \mathrm{~min}$, which supports previous literature suggesting the condition is short-lived and occurs within the first $30 \mathrm{~min}$ of anesthesia emergence $[19,20]$. Although EA is a time-limited phenomenon with no clinical evidence of negative long-term effects, it may lead to higher complication rates. In addition, it can be very distressing for children and parents alike. Therefore, concerns about emergence behaviors in children undergoing sevoflurane anesthesia remain, although sevoflurane is a useful pediatric anesthetic that does not irritate the airway and is associated with hemodynamic stability and rapid induction and emergence. Preoperative anxiety is one of the proposed contributing factors to EA [22]. Midazolam, a widely used anxiolytic agent, was investigated as a medication for preventing EA. However, a meta-analysis examining the pharmacologic prevention of EA showed that midazolam had no effects on incidence of EA [23]. In addition, parental presence in the operating room alleviates preoperative anxiety and may prevent EA [24]. In the present study, there were no significant differences in anxiety scores at the ward and operating room between two groups.

EA has been shown to be associated with postoperative pain. The effectiveness of analgesic drugs such as fentanyl, $\alpha_{2}-$ agonists, and ketamine on the treatment or prevention of EA could be explained by the fact that they alleviate postoperative pain. However, pain can be difficult to assess in preverbal children and may mimic the signs of EA, making it difficult to differentiate EA from postoperative pain. Although intravenous $[10,25]$ or intranasal fentanyl [9] and dexmedetomidine $[26,27]$ are effective in treating both pain and EA, the exact role of pain in the etiology of EA remains unclear. Although ketamine, fentanyl, and dexmedetomidine are known medications in preventing EA, a subgroup analysis of preoperative analgesia found that their analgesic effects had no influence on the efficacy of the drugs on EA [23]. Additionally, the type of surgery or method of intraoperative pain management did not alter the risk for EA [7]. Furthermore, EA can also occur in pain-free children $[8,15]$ and the incidence of EA is similar in painful and non-painful procedures, which supports the conclusion that pain does not contribute to EA $[2,3,5]$. These results suggest that the analgesic properties of these compounds are unlikely to contribute to their preventive effects.

Keidan et al. [28] compared the incidence of postoperative nausea and vomiting (PONV) and 48-h recovery profiles after a single dose of ketorolac vs. fentanyl in dexamethasonepretreated children undergoing ambulatory adenoidectomy. They reported that the incidence of PONV and postoperative pain scores were equal in both groups. However, agitation scores in the PACU were significantly higher in the ketorolac 
group. Although the study investigated the effect of ketorolac on agitation scores, the main purpose of the study was to examine PONV and postoperative pain, and the study did not compared the incidence and severity of EA with the control group. In the present study, the control group did not receive any preoperative or intraoperative analgesics and the ketorolac group received $1 \mathrm{mg} / \mathrm{kg}$ of ketorolac at the end of surgery. Most factors of pediatric pain scales (e.g., face legs activity cry and consolability (FLACC) pain/discomfort scale), such as crying, movement, posture, and consolability, were also parts of agitation scales. The authors were concerned that the agitation scores could be affected by the pain score measurement, which could be a critical bias. Therefore, we did not measure the pain score, but the attending anesthesiologist in PACU administered fentanyl intravenously and recorded the amount and the time when the patient verbally complained of pain or behaved in manner consistent with being in pain. There was a statistically significant difference in the number of patients who received fentanyl for analgesia after $20 \mathrm{~min}$ in the PACU between the two groups $(13 / 39,33 \%$ in the control group and $5 / 41,12 \%$ in the ketorolac group; $\mathrm{P}=0.046$ ). If postoperative pain contributed to the incidence and severity of EA, the number of patients with EA and agitation scores should be higher in the control group than the ketorolac group. However, there were no differences in the incidence and severity of EA between two groups. The results support the view that the analgesic effect of ketorolac does not affect the incidence and severity of EA.

There was a critical limitation in studies of EA including this study. Because there are various EA assessment tools and definitions and the difficulties in interpreting behavior, observational differences result in a wide range of estimates of EA in children recovering from general anesthesia from as low as $25 \%$ to as high as $80 \%$. This study may be criticized for the potential effects of pain on the assessment of EA. Because it can be difficult to differentiate EA from postoperative pain, and pain can affect EA scores, the authors did not assess postoperative pain. The authors believed that EA could be distinguished from postoperative pain in this study because children aged $3-7$ years old are verbal and can describe their pain. However, it is difficult to exclude the effect of pain on the severity and incidence of EA. Several types of surgical procedures including tonsillectomy were allocated in this study, which is another limitation. The different nature of postoperative pain from various surgical procedures can affect the EA. Because there was no significant difference between two groups, it may not affect the incidence of EA. Ketorolac is recommended to use cautiously in tonsillectomy patients because of postoperative bleeding, but it is still controversial $[29,30]$. In the author's institution, NSAIDs including ketorolac are more commonly used for postoperative pain control in pediatric patients after tonsillectomy than opioids.

In conclusion, the results of this study show that the administration of $1 \mathrm{mg} / \mathrm{kg}$ of ketorolac at the end of surgery was not effective in decreasing the incidence and severity of EA in 3-7-year-old children undergoing sevoflurane anesthesia. This result supports the view that the EA is not prevented by NSAIDs. Therefore, other interventions should be considered for prevention of EA in high-risk children.

\section{References}

1. Sikich N, Lerman J. Development and psychometric evaluation of the pediatric anesthesia emergence delirium scale. Anesthesiology 2004; 100: 1138-45.

2. Voepel-Lewis T, Malviya S, Tait AR. A prospective cohort study of emergence agitation in the pediatric postanesthesia care unit. Anesth Analg 2003; 96: 1625-30.

3. Uezono S, Goto T, Terui K, Ichinose F, Ishguro Y, Nakata Y, et al. Emergence agitation after sevoflurane versus propofol in pediatric patients. Anesth Analg 2000; 91: 563-6.

4. Welborn LG, Hannallah RS, Norden JM, Ruttimann UE, Callan CM. Comparison of emergence and recovery characteristics of sevoflurane, desflurane, and halothane in pediatric ambulatory patients. Anesth Analg 1996; 83: 917-20.

5. Cravero J, Surgenor S, Whalen K. Emergence agitation in paediatric patients after sevoflurane anaesthesia and no surgery: a comparison with halothane. Paediatr Anaesth 2000; 10: 419-24.

6. Picard V, Dumont L, Pellegrini M. Quality of recovery in children: sevoflurane versus propofol. Acta Anaesthesiol Scand 2000; 44: 30710.

7. Cole JW, Murray DJ, McAllister JD, Hirshberg GE. Emergence behaviour in children: defining the incidence of excitement and agitation following anaesthesia. Paediatr Anaesth 2002; 12: 442-7.

8. Cravero JP, Beach M, Thyr B, Whalen K. The effect of small dose fentanyl on the emergence characteristics of pediatric patients after sevoflurane anesthesia without surgery. Anesth Analg 2003; 97: 364-7.

9. Finkel JC, Cohen IT, Hannallah RS, Patel KM, Kim MS, Hummer KA, et al. The effect of intranasal fentanyl on the emergence characteristics after sevoflurane anesthesia in children undergoing surgery for bilateral myringotomy tube placement. Anesth Analg 2001; 92: 11648.

10. Cohen IT, Hannallah RS, Hummer KA. The incidence of emergence agitation associated with desflurane anesthesia in children is reduced by fentanyl. Anesth Analg 2001; 93: 88-91.

11. Ozer Z, Gorur K, Altunkan AA, Bilgin E, Camdeviren H, Oral U. Efficacy of tramadol versus meperidine for pain relief and safe recovery after adenotonsillectomy. Eur J Anaesthesiol 2003; 20: 920-4.

12. Buckley MM, Brogden RN. Ketorolac. A review of its pharmacodynamic and pharmacokinetic properties, and therapeutic potential. Drugs 1990; 39: 86-109.

13. Lieh-Lai MW, Kauffman RE, Uy HG, Danjin M, Simpson PM. A randomized comparison of ketorolac tromethamine and morphine for postoperative analgesia in critically ill children. Crit Care Med 1999; 27: 2786-91. 
14. Kain ZN, Mayes LC, Cicchetti DV, Bagnall AL, Finley JD, Hofstadter MB. The Yale Preoperative Anxiety Scale: how does it compare with a "gold standard"? Anesth Analg 1997; 85: 783-8.

15. Aono J, Ueda W, Mamiya K, Takimoto E, Manabe M. Greater incidence of delirium during recovery from sevoflurane anesthesia in preschool boys. Anesthesiology 1997; 87: 1298-300.

16. Voepel-Lewis T, Burke C, Hadden SM, Tait AR, Malviya S. Nurses' diagnoses and treatment decisions regarding care of the agitated child. J Perianesth Nurs 2005; 20: 239-48.

17. Wells LT, Rasch DK. Emergence “delirium” after sevoflurane anesthesia: a paranoid delusion? Anesth Analg 1999; 88: 1308-10.

18. Lapin SL, Auden SM, Goldsmith LJ, Reynolds AM. Effects of sevoflurane anaesthesia on recovery in children: a comparison with halothane. Paediatr Anaesth 1999; 9: 299-304.

19. Hudek K. Emergence delirium: a nursing perspective. AORN J 2009; 89: 509-16.

20. Vlajkovic GP, Sindjelic RP. Emergence delirium in children: many questions, few answers. Anesth Analg 2007; 104: 84-91.

21. Malarbi S, Stargatt R, Howard K, Davidson A. Characterizing the behavior of children emerging with delirium from general anesthesia. Paediatr Anaesth 2011; 21: 942-50.

22. Kain ZN, Caldwell-Andrews AA, Maranets I, McClain B, Gaal D, Mayes LC, et al. Preoperative anxiety and emergence delirium and postoperative maladaptive behaviors. Anesth Analg 2004; 99: 164854.

23. Dahmani S, Stany I, Brasher C, Lejeune C, Bruneau B, Wood C, et al. Pharmacological prevention of sevoflurane- and desflurane-related emergence agitation in children: a meta-analysis of published studies. Br J Anaesth 2010; 104: 216-23.

24. Arai YC, Ito H, Kandatsu N, Kurokawa S, Kinugasa S, Komatsu T. Parental presence during induction enhances the effect of oral midazolam on emergence behavior of children undergoing general anesthesia. Acta Anaesthesiol Scand 2007; 51: 858-61.

25. Jung HJ, Kim JB, Im KS, Oh SH, Lee JM. Effect of ketamine versus thiopental sodium anesthetic induction and a small dose of fentanyl on emergence agitation after sevoflurane anesthesia in children undergoing brief ophthalmic surgery. Korean J Anesthesiol 2010; 58: 148-52.

26. Isik B, Arslan M, Tunga AD, Kurtipek O. Dexmedetomidine decreases emergence agitation in pediatric patients after sevoflurane anesthesia without surgery. Paediatr Anaesth 2006; 16: 748-53.

27. Patel A, Davidson M, Tran MC, Quraishi H, Schoenberg C, Sant $\mathrm{M}$, et al. Dexmedetomidine infusion for analgesia and prevention of emergence agitation in children with obstructive sleep apnea syndrome undergoing tonsillectomy and adenoidectomy. Anesth Analg 2010; 111: 1004-10.

28. Keidan I, Zaslansky R, Eviatar E, Segal S, Sarfaty SM. Intraoperative ketorolac is an effective substitute for fentanyl in children undergoing outpatient adenotonsillectomy. Paediatr Anaesth 2004; 14: 318-23.

29. Rømsing J, Ostergaard D, Walther-Larsen S, Valentin N. Analgesic efficacy and safety of preoperative versus postoperative ketorolac in paediatric tonsillectomy. Acta Anaesthesiol Scand 1998; 42: 770-5.

30. Agrawal A, Gerson CR, Seligman I, Dsida RM. Postoperative hemorrhage after tonsillectomy: use of ketorolac tromethamine. Otolaryngol Head Neck Surg 1999; 120: 335-9. 